\title{
Redes de seguridad para cierre vertical de fachadas
}

\section{Vertical safety nets closing façade gaps}

\author{
P. Riquelme ${ }^{(*)}$, S. Romero ${ }^{(* *)}, \underline{\text { R. Irles }}{ }^{(*)}$, E. Segovia ${ }^{(*)}$
}

\section{RESUMEN}

Entre otros medios de protección colectiva, en los últimos años se vienen utilizando en España redes verticales de cierre de fachadas, a paño completo entre forjados en fase de estructura, y también para huecos menores como ventanas o puertas, en fases posteriores. Este tipo de protección no cuenta hasta el momento con ningún tipo de regulación oficial, si bien en el grupo de trabajo AEN/CTN81/SC2/GT7 se desarrolla actualmente el borrador de la norma Pr UNE 81651 a tal fin. Desde el $\mathrm{Dt}^{\circ}$. de Ing. de la Construcción de la Universidad de Alicante, varios miembros del grupo de trabajo han desarrollado, contando con la experiencia en otros sistemas de protección, algunos modelos numéricos con elementos finitos para valorar y cuantificar variables mecánicas asociadas al episodio de una eventual caída de una persona sobre este tipo de redes, caracterizado por una menor energía cinética que para otros tipos de ellas. En el artículo se exponen los resultados obtenidos para ambos tipos de paños, grandes y pequeños, donde se han valorado la separación de anclajes de la red, las aberturas generadas en su perímetro durante la retención, los esfuerzos en anclajes y elementos textiles, el factor de impacto que sufriría el accidentado y la repercusión de la disposición geométrica de la red, al cuadro o al rombo.

Palabras clave: Red; seguridad; fachada; caída; impacto.

\section{SUMMARY}

Among other methods, vertical safety nets on the façades of buildings during the construction stage are used in Spain as an alternative to other safety systems in order to avoid falls to a different level. There are two kinds of such nets: floorto-ceiling nets - that secure the entire void between successive floors in structure construction stage-, and small nets what secure windows and other minor voids in later stages. Their design and requirements are not defined at present, but the AEN/CTN81/SC2/GT7 Spanish Working Group is drafting a specific regulation named Pr UNE 81651. Some members of the Civil Engineering Department of the University of Alicante, having experience in other systems of protection, have developed several finite-elements numerical models to measure and analyse mechanical variables related to a possible fall of a person over this kind of net, characterised by a kinetic energy lower than for other types. In this paper the obtained results are shown, for both large and little nets, analysing the anchorages gap, void openings at perimeter, maximum net deflection and net disposal (square/diamond) repercussion, as well as its consequences on the victim's safety (impact factor).

Keywords: Net; safety; façade; fall; impact.

(*) Universidad de Alicante (España).

${ }^{(* *)}$ CONSOMAR, S.A. Valencia (España).

Persona de contacto/Corresponding author: ramon.irles@ua.es (R. Irles)

Cómo citar este artículo/Citation: Riquelme, P., Romero, S., Irles, R., Segovia, E. (2014). Redes de seguridad para cierre vertical de fachadas. Informes de la Construcción, 66(533): eoo2, doi: http://dx.doi.org/10.3989/ic.11.132.

Licencia / License: Salvo indicación contraria, todos los contenidos de la edición electrónica de Informes de la Construcción se distribuyen bajo una licencia de uso y distribución Creative Commons Reconocimiento no Comercial 3.o. España (cc-by-nc). 


\section{INTRODUCCIÓN}

Preservar la seguridad e higiene en el trabajo viene establecido como un deber constitucional (artículo 40.2 de la Constitución Española) de los poderes públicos. La presencia de España en la Unión Europea genera la necesidad de armonizar nuestra política con la creciente política comunitaria en todas las materias, y en particular en la prevención de los riesgos derivados del trabajo (transposición de la Directiva 89/391/CEE) (1). Junto a este marco jurídico, se encuentran nuestros compromisos contraídos con la Organización Internacional del Trabajo a partir de la ratificación del Convenio 155 sobre seguridad y salud de los trabajadores y medio ambiente de trabajo. Asimismo, España tiene que hacer frente a la alta siniestralidad en el sector de la construcción con cifras que según fuentes del Ministerio de Trabajo (Análisis de la mortalidad por accidente de trabajo en España - 2005-2007) supera las quinientas muertes (516) investigadas, correspondiéndose el 17,25\% de ellas con la actividad Estructuras y obras de fábrica, el 13,37\% con la actividad Cerramientos externos y el 6,98\% y 6,59\% con Acabados y Cubiertas, respectivamente. En conjunto, estas actividades representan el 44,19\%, y en todas ellas «se observa que la circunstancia anómala (desviación) que ha causado más fallecidos en el sector de la Construcción es la Caída de personas $(32,75 \%) »$.

Estas actividades nombradas son propias de la edificación, y ésta aglutina el 66,09\% de los accidentes mortales investigados. Cumpliendo con la normativa estatal (2) y (3), las caídas de altura desde los huecos y aberturas existentes en los pisos de las obras, que supongan para los trabajadores un riesgo de caída de altura superior a 2 metros, se protegerán mediante barandillas u otro sistema de protección colectiva. Este otro sistema de protección colectiva, una vez regulado, ensayado y probado, pueden ser las redes de seguridad verticales en fachadas.

La norma UNE-EN 1263, (4) y (5) referente a las Redes de Seguridad, contempla los requisitos de seguridad y los métodos de ensayo para las redes de seguridad homologadas. Las redes recogidas por esta normativa son de cuatro tipos de sistema de protección:

A. Sistema S: Red de Seguridad con cuerda perimetral.

B. Sistema T: Red de Seguridad sujeta a consolas para su utilización horizontal.

C. Sistema U: Red de Seguridad sujeta a una estructura soporte para su utilización vertical.

D. Sistema V: Red de Seguridad con cuerda perimetral sujeta a un soporte tipo horca.

En la descripción de los métodos de ensayo se especifica que la muestra debe tener unas dimensiones de $(3 \pm 0,1) \mathrm{m} \times$ $(3 \pm 0,1) \mathrm{m}$ para sistemas tipo $\mathrm{S}$, similar a las redes de seguridad verticales en fachadas.

Las redes de cierre vertical en fachada se utilizan en España como alternativa a otros sistemas de seguridad, como las redes tipo V (4) y (5) o las barandillas (6), para evitar caídas a diferente nivel. Podemos considerar dos clases de estas redes: grandes y pequeñas. Las primeras cierran normalmente todo el paño de suelo a techo cuando no se han ejecutado los cerramientos; las segundas cierran huecos menores como puertas, ventanas u otros, ejecutados ya los tabiques de ce- rramiento pero antes de instalar la cerrajería. El tamaño de la red resulta importante, ya que las redes pequeñas sujetas por su perímetro a puntos fijos resultan apreciablemente más rígidas que las grandes.

Este tipo de redes previene caídas de altura reteniendo al posible accidentado con menor energía cinética que otras, como las de tipo $\mathrm{V}$ o las barandillas tipo $\mathrm{B}$ y $\mathrm{C}$, ya que la pérdida de cota hasta el impacto viene limitada a la posible por la caída dentro del mismo piso, y generalmente esto la limita a unos $2 \mathrm{~m}$. Con ello, la velocidad de incidencia sobre la red resulta de unos $6,3 \mathrm{~m} / \mathrm{s}$. Los criterios para su diseño y requisitos a cumplir no están definidos actualmente en ningún texto normativo, si bien el grupo de trabajo AEN/ CTN81/SC2/GT7 se ocupa desde hace meses de preparar un borrador de norma. Inspirado en los requisitos de los ensayos de (4) y (5), el grupo está considerando el requisito de superación de una prueba con lastre esférico de $100 \mathrm{Kg}$ cayendo desde dicha altura contra la red. Del objetivo último de la protección del potencial accidentado, resulta el criterio básico de conseguir un impacto (es decir, el esfuerzo máximo desarrollado sobre el cuerpo durante la retención) suficientemente bajo.

Para predecir y evaluar las variables geométricas y mecánicas del fenómeno dinámico de la retención, se han analizado varios modelos numéricos mediante elementos finitos, con base en las investigaciones que varios de los autores han desarrollado en otros dispositivos similares durante los últimos 20 años (7) (8) (9) (10) (11) (12) (13) (14) (15) (16) (17), simulando la caída de tal lastre sobre la red. Los modelos analizados y los resultados obtenidos se describen a continuación para ambos tamaños de red.

\section{ESTUDIOS REALIZADOS}

Las redes verticales de seguridad estudiadas corresponden al Tipo de Cierre Vertical (Redes de seguridad colocadas en fachada y sujetas a la estructura).

Este tipo de red se utiliza generalmente en edificación, para proceder al cierre del perímetro de la estructura en ausencia de fachada (Figura 1) o con la terminación parcial de ésta, hasta que se puedan establecer las condiciones de seguridad que permitan su retirada.

Su sujeción se viene realizando a los forjados superior e inferior mediante anclaje mecánico con un máximo de o,50 m de separación, aunque en ocasiones se realiza mediante piezas auxiliares sujetas a la armadura, previo hormigonado del forjado. En los laterales, las redes se unen entre sí a través del cordón textil que forma el perímetro de la red (cuerda de unión), pudiéndose sujetar directamente a los pilares si no presenta continuidad. En aquellos lugares donde no se dispusiera cuerda perimetral, la red suele sujetarse a la estructura soporte malla a malla.

El material utilizado para confeccionar la red está compuesto por productos sintéticos como la poliamida o el polipropileno de alta tenacidad, formando con las cuerdas una malla anudada o tejida de $10 \mathrm{~cm}$, cuadrada o en rombo, y con una energía de rotura mínima de $2,3 \mathrm{~kJ}$ (4). La cuerda perimetral de seguridad será como mínimo de $10 \mathrm{~mm}$ de diámetro y $30 \mathrm{kN}$ de resistencia, y los módulos de red serán atados entre sí con cuerda de poliamida o poliéster como mínimo de $3 \mathrm{~mm}$. 


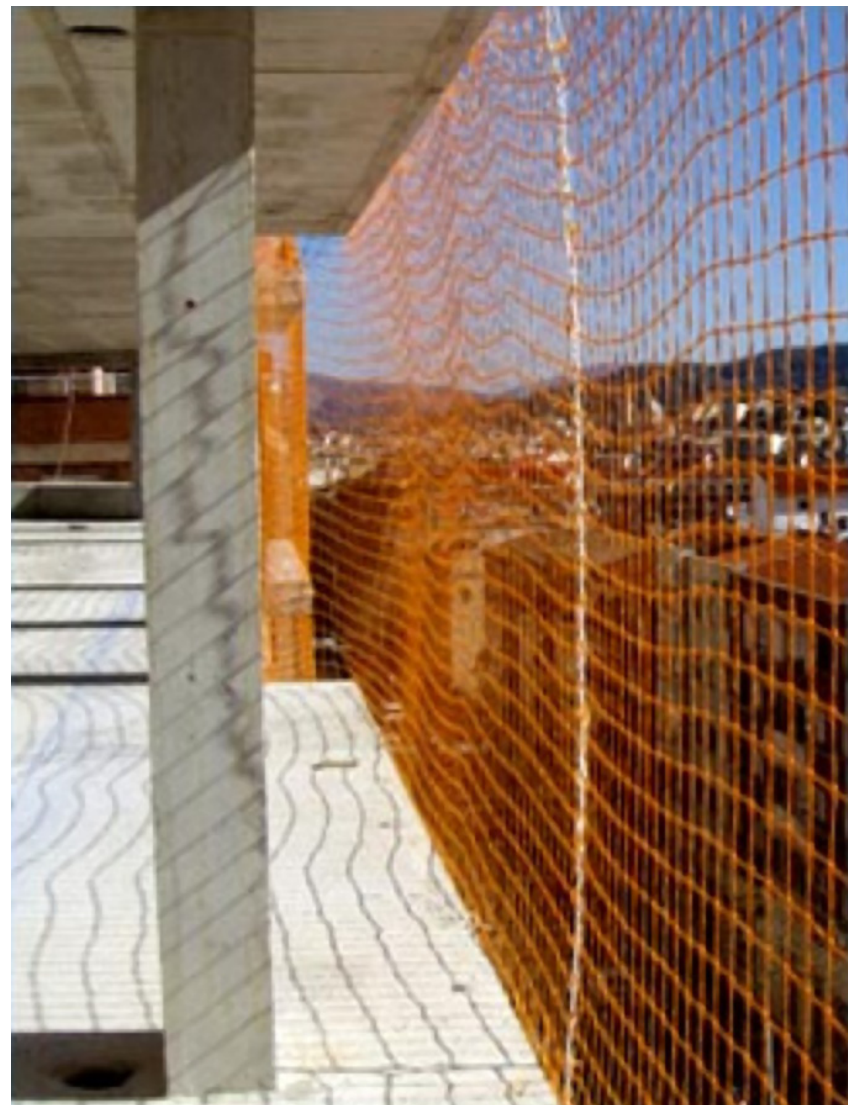

Figura 1. Red de cierre vertical colocada en obra.

\subsection{Modelo numérico}

Se han realizado dos tipos de modelos, uno para redes pequeñas y otro para redes grandes (10) (11), (13).

En los modelos para redes pequeñas de $1 \times 1 \mathrm{~m}^{2}$ se ha estudiado la influencia sobre las medidas controladas de los siguientes parámetros:

- Geometría de la malla,

- Rigidez de la trencilla,

- Excentricidad de la carga,

- Cuerdas perimetrales y

- Deformación inicial.

Asimismo, para el caso de redes grandes, mediante el modelo de elementos finitos se ha estudiado una red de tamaño $4 \times 3 \mathrm{~m}^{2}$, con malla cuadrada de $10 \times 10 \mathrm{~cm}^{2}$, sujeta por su perímetro conforme a las siguientes alternativas:

a) Cada $50 \mathrm{~cm}$ en sus lados superior e inferior, quedando sus laterales libres.

b) Cada $50 \mathrm{~cm}$ en todo el perímetro.

c) Cada $100 \mathrm{~cm}$ en todo el perímetro.

d) Cada $100 \mathrm{~cm}$ en sus lados superior e inferior, quedando sus laterales libres.

En ambos tipos de redes, el lastre se ha tomado esférico de $100 \mathrm{~kg}$ de masa y $50 \mathrm{~cm}$ de diámetro, incidiendo normalmente al plano inicial de la red, con velocidad correspondiente a $2 \mathrm{~m}$ de caída. Para la redes grandes, el impacto sobre la red se ha producido sobre el cuarto de la malla inferior izquierda, de tal forma que se simulen 12 impactos en cuadrícula de $50 \times 50 \mathrm{~cm}^{2}$, tal y como se indica en la Figura 2, por cada tipo de red.

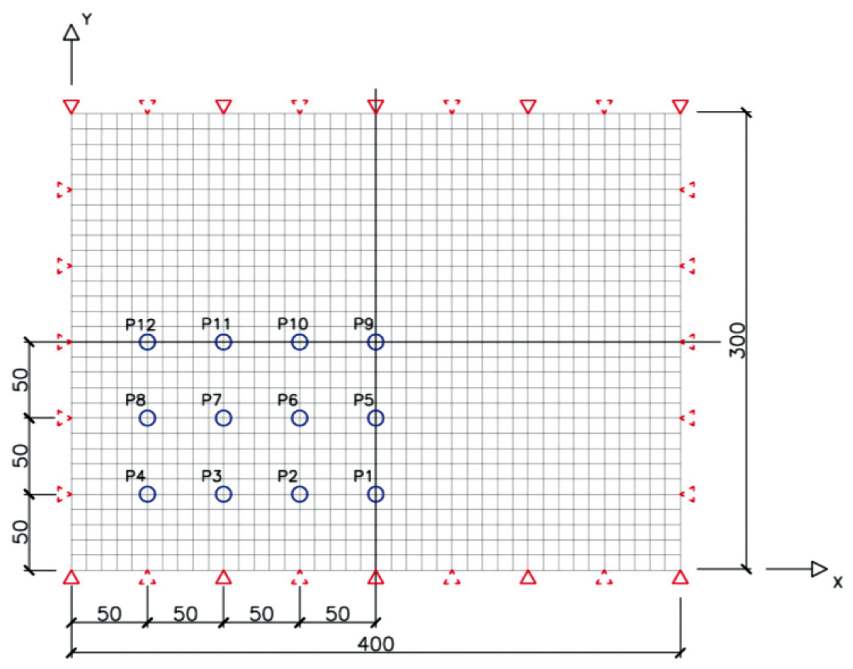

Figura 2. Impactos del lastre en la red grande y dimensiones.

Las medidas de los resultados a controlar en cada uno de los modelos son:

- Esfuerzos en los anclajes producidos por los impactos ( Fx, $\mathrm{Fy}, \mathrm{Fz}$ ).

- Flecha máxima producida en la red.

- Factor de impacto (aceleración máxima instantánea / g).

- Desplazamiento horizontal de la cuerda perimetral vertical.

- Desplazamiento de las trencillas entre anclajes (abertura del perímetro inferior).

- Esfuerzos en trencillas y cuerda perimetral.

Los modelos se han desarrollado con el software de Elementos Finitos ANSYS, utilizando las siguientes capacidades para la recreación del modelo:

- Análisis dinámico transitorio.

- Comportamiento a tracción de la red elástico lineal hasta rotura, con amortiguamiento, sin rigidez a compresión.

- Desarrollo de grandes movimientos y giros, llegando a presentarse en la red grandes deformaciones.

- Rigidización por tensión en la red.

- Contacto variable entre lastre y red.

En cuanto al modelo sólido, la red se representa mediante elementos lineales articulados en sus extremos (o sea, sin características de flexión), con una la ley de comportamiento simplificada bilineal (sin resistencia a compresión) capaz de recoger, de forma «equivalente», la capacidad de absorción de energía de los nudos.

Dicha equivalencia fue ajustada con los resultados experimentales disponibles para redes tipo $\mathrm{V}$ obtenidos por Segovia, (8) y (15), asumiéndose su validez para el tipo de redes estudiado en este trabajo a falta de ensayos reales que lo confirmen.

Esta ley de comportamiento bilineal para la red se aleja de la real (19) principalmente por dos motivos:

1. El apriete de los nudos, cuyo efecto sobre la absorción de energía en nuestro modelo viene recogido por el amortiguamiento estructural $(\beta)$ y cuyo efecto sobre la mayor deformabilidad aparente se recoge en el valor ficticio de $k_{\mathrm{r}}$ (rigidez a tracción de las trencillas). 
2. Las grandes deformaciones que aparecen en la red, que provocan que la relación entre tensión y deformación cuando la red trabaja a tracción no sea una línea recta.

Pero se adopta en el análisis, puesto que las calibraciones para redes tipo V mostraron que, en su conjunto, se comporta de forma equivalente a la observada en los ensayos experimentales, y las experiencias en curso para otros sistemas parecen confirmar su validez.

El material que compone las trencillas de la red fue modelado con elementos LINK10 con comportamiento viscoelástico $\beta=0,34$, con una masa de $0,008 \mathrm{~kg} / \mathrm{m}$ y una rigidez $\mathrm{kr}=610 \mathrm{~N}$.

Por economía en el cálculo y por encontrarse los mayores esfuerzos durante la fase transitoria del análisis dinámico, se puede tratar de forma equivalente el comportamiento del material ajustando la rigidez de forma que la deformación plástica, además de la capacidad de absorción de energía de los nudos, quede reflejada con un modelo lineal equivalente del material. La deformación plástica quedaría para la fase permanente y ésta no es objeto de estudio. Tampoco conocemos cuánta energía se pierde en el «apriete» de los nudos por lo que la rigidez real de la red se ajustará con el modelo con una ficticia. No parece desproporcionado adoptar un modelo lineal para el material.

\subsection{Simulaciones realizadas}

Para el estudio de las redes grandes mediante análisis numérico con el modelo expuesto, se ha procedido al diseño y cálculo de 48 modelos con 12 posiciones de impacto por cada tipo de red grande. Los resultados se han agrupado en 4 bloques, según sea el apoyo perimetral:

- Bloque 1: Red sujeta cada 50 centímetros en sus lados superior e inferior, quedando sus laterales libres y con una cuerda textil formando el perímetro lateral (Ensayos 1-12).

- Bloque 2: Red sujeta cada 50 centímetros en todo el perímetro, con cuerda textil perimetral (Ensayos 13-24).

- Bloque 3: Red sujeta cada 100 centímetros en sus lados superior e inferior, quedando sus laterales libres y con una cuerda textil formando el perímetro lateral (Ensayos 25-36).

- Bloque 4: Red sujeta cada 100 centímetros en todo el perímetro, con cuerda textil perimetral (Ensayos 37-48).

En cuanto a las redes pequeñas, se ha realizado igualmente la modelización del impacto de un lastre de $100 \mathrm{~kg}$ en caída libre desde $2 \mathrm{~m}$ de altura. La red que sufre el impacto es cuadrada de $1,0 \mathrm{~m} \times 1,0 \mathrm{~m}$ con tamaño de malla de $10 \mathrm{~cm}$, anclada únicamente en sus cuatro extremos. La modelización de los anclajes no se ha realizado, considerando éstos con rigidez suficiente. Se han analizado las dos disposiciones posibles: con red al cuadro (Q) o al rombo (D), Figura 3.

Se han considerado todos los elementos de la red como puntos de contacto potencial con la esfera, dadas las dimensiones de ambas. Con impacto centrado sólo se verían afectados los elementos centrales de la red pero con excentricidad se puede dar un desplazamiento de la esfera en la red, obligando a aumentar el número de elementos de contacto.

Para el estudio de las redes pequeñas se han analizado varios tipos de modelos, modificando en cada uno de ellos una o dos variables y analizando la evolución o sensibilidad relativa.
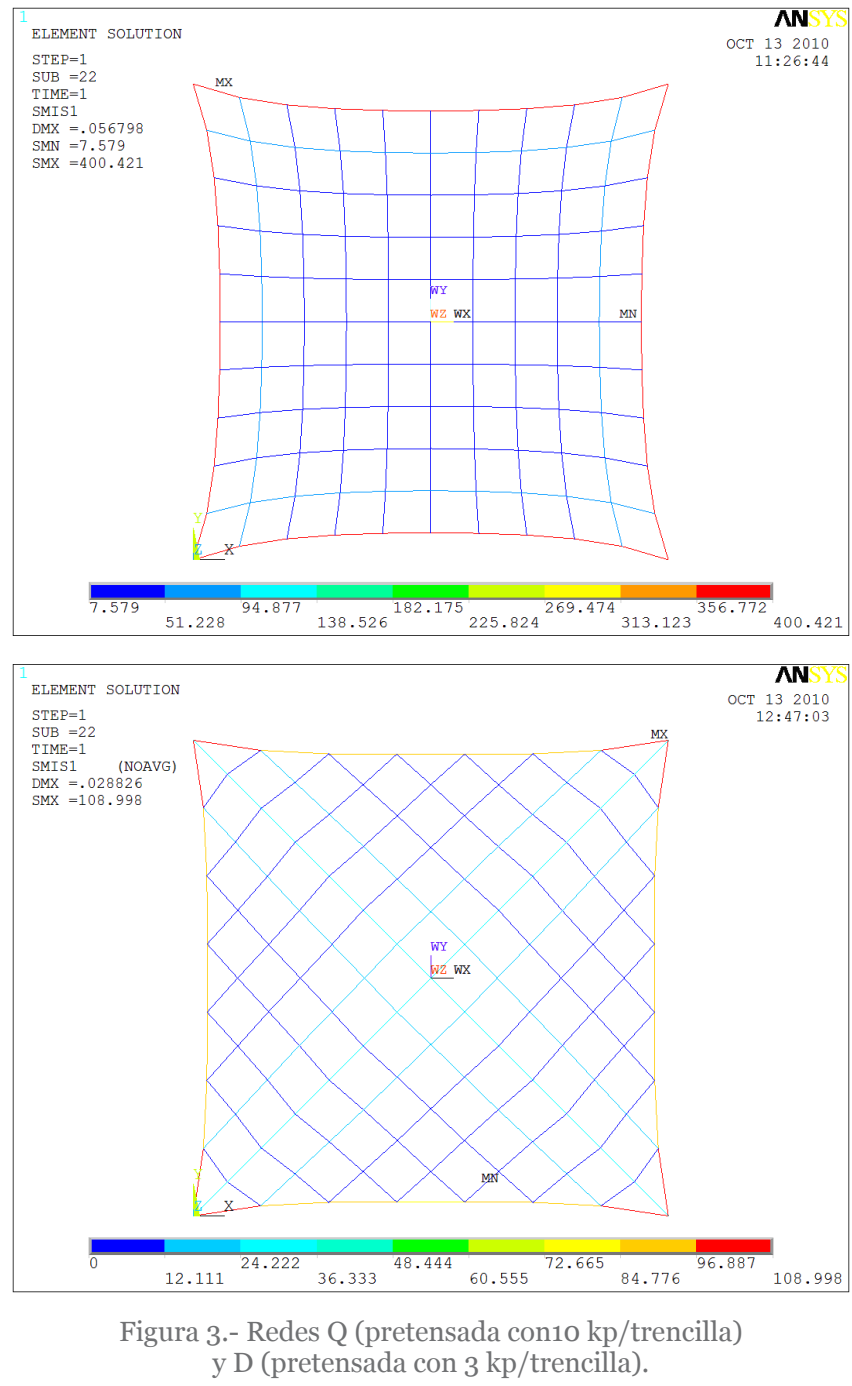

Se ha estudiado la influencia sobre las medidas controladas, descritas en 2.1, de los siguientes parámetros:

- Geometría de la malla: malla cuadrada (Q) o malla al rombo (D).

- Rigidez de la trencilla: variando desde $400 \mathrm{~N}$ a $2000 \mathrm{~N}$.

- Excentricidad de la carga: con posiciones centradas y excéntricas.

- Cuerdas perimetrales: con cuerdas textiles en todo el perímetro y con cables de acero en los bordes horizontales.

- Tensión inicial de la red: producida por una fuerza de hasta $20 \mathrm{~kg} /$ trencilla.

\section{RESULTADOS}

Del análisis de los resultados de las medidas controladas, se obtienen las conclusiones derivadas del análisis de los modelos numéricos. Dividiremos éstos por tipo de red, de paño grande y de paño pequeño.

\subsection{Redes de paño grande}

\subsubsection{Reacciones en anclajes}

La colocación de la red con anclajes laterales produce una reducción significativa de las reacciones frente a la colocación con laterales libres. Esto se hace notar más cuanto más cerca- 
nos están dispuestos, pudiendo alcanzar valores de reducción de la resultante del 50\%.

En el caso de los anclajes con 1 metro de separación, la presencia de los anclajes laterales lleva el punto de reacción máxima a la esquina. Esto puede ser debido a la menor superficie de red para el reparto de esfuerzos unido a la elevada distancia de separación entre anclajes, lo cual hace que el anclaje de esquina no pueda repartir el esfuerzo con los anclajes cercanos.

El mayor valor de la resultante de la reacción es de $2377 \mathrm{~N}$, obtenido para impacto en posición 8 (Figura 2), con laterales libres y anclajes a $100 \mathrm{~cm}$ (ensayo $\left.\mathrm{n}^{0} 32\right)$.

Respecto a la composición de la reacción en los anclajes, en los casos con laterales libres es la componente $F y$ la que absorbe más fuerza. En el caso de la red anclada en sus cuatro bordes, la componente $\mathrm{Fz}$ es la preponderante en la resultante del anclaje. Es decir, la red anclada en todo el perímetro incrementa las reacciones en el plano de la red reduciendo la resultante. La Tabla 1 muestra a continuación los máximos valores de la resultante en cada modelo modelo.

Tabla 1.

Valores de reacción máxima según modelo.

\begin{tabular}{|c|c|}
\hline No ENSAYO & F(N) \\
\hline 4 & 1730,1 \\
\hline 8 & 2018,58 \\
\hline 12 & 2057,91 \\
\hline 14 & 947,96 \\
\hline 20 & 1006,62 \\
\hline 21 & 689,24 \\
\hline 24 & 1002,06 \\
\hline 28 & 2182,27 \\
\hline 32 & 2376,95 \\
\hline 36 & 2375,33 \\
\hline 40 & 1841,58 \\
\hline 43 & 1100,6 \\
\hline 44 & 1531,39 \\
\hline
\end{tabular}

El punto de impacto que crea mayores reacciones también cambia, pero en ello influye además la separación de los anclajes. En anclajes separados $50 \mathrm{~cm}$, la presencia de anclajes laterales produce un aumento de la franja donde se producen las reacciones máximas, pasando de ser el punto central del lateral a una franja de 1 metro de longitud a lo largo de ese lateral. Esto se puede apreciar en el Mapa de impactos con valores de reacción máxima que se muestra en la Figura 4.

Los puntos de impacto con valores de flecha máxima y mínima son el centro de la red y la esquina, respectivamente, en todos los casos. El valor de la flecha máxima es de $1,40 \mathrm{~m}$, obtenido para el impacto centrado, separación de anclajes de $100 \mathrm{~cm}$ y laterales libres. La flecha mínima es de 0,74 m, obtenido para el impacto en esquina, separación $50 \mathrm{~cm}$ y laterales anclados.

\subsubsection{Flecha máxima}

Independientemente de la separación de anclajes, su disposición en los laterales de la red produce una reducción muy baja de los valores de flecha obtenidos (del orden de $10 \mathrm{~cm}$ ) y un aumento en la diferencia entre sus valores máximo y mínimo variando la excentricidad del impacto. Si bien con separación de $100 \mathrm{~cm}$ los valores obtenidos son unos $10 \mathrm{~cm}$ superiores a los que aparecen con $50 \mathrm{~cm}$ de separación.

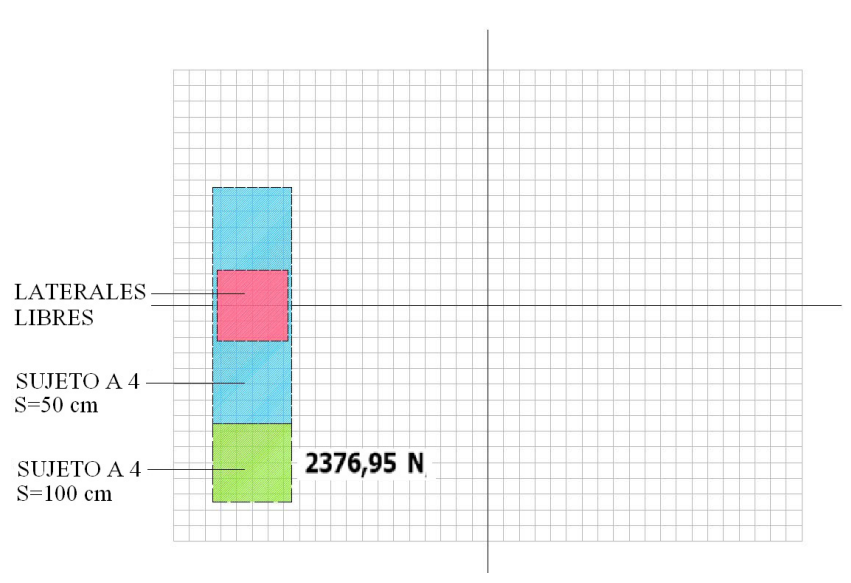

Figura 4. Mapa de impactos con valores de reacción máxima

En la Tabla 2 se muestran los valores de las flechas máximas alcanzadas en cada modelo.

Analizando la Tabla 2 se pueden observar las variaciones en la flecha para el mismo punto de impacto (misma columna) producidas por los cuatro tipos de contornos (misma fila). El bloque 3 con laterales libres y distancia entre anclajes de $100 \mathrm{~cm}$, muestra siempre las mayores flechas. Por el contrario, el bloque 2, con red sujeta en todo el perímetro cada 50 $\mathrm{cm}$, muestra siempre las menores flechas. En las Figura 5 y

Tabla 2.

Valores de flecha máxima según modelo.

\begin{tabular}{|c|c|c|c|c|c|c|c|c|c|c|c|c|}
\hline TIPO ENSAYO & $\mathbf{1}$ & 2 & 3 & 4 & 5 & 6 & 7 & 8 & 9 & 10 & 11 & 12 \\
\hline $\begin{array}{l}\text { FLECHA } \\
\text { MÁXIMA (metros) }\end{array}$ & $-0,993$ & $-0,987$ & $-0,984$ & $-0,939$ & $-1,197$ & $-1,192$ & $-1,181$ & $-1,122$ & $-1,261$ & $-1,257$ & $-1,241$ & $-1,178$ \\
\hline TIPO ENSAYO & 13 & 14 & 15 & 16 & 17 & 18 & 19 & 20 & 21 & 22 & 23 & 24 \\
\hline $\begin{array}{l}\text { FLECHA } \\
\text { MÁXIMA (metros) }\end{array}$ & $-0,907$ & $-0,897$ & $-0,845$ & $-0,736$ & $-1,082$ & $-1,064$ & $-1,002$ & $-0,849$ & $-1,132$ & $-1,107$ & $-1,048$ & $-0,876$ \\
\hline TIPO ENSAYO & 25 & 26 & 27 & 28 & 29 & 30 & 31 & 32 & 33 & 34 & 35 & 36 \\
\hline $\begin{array}{l}\text { FLECHA } \\
\text { MÁXIMA (metros) }\end{array}$ & $-1,108$ & $-1,142$ & $-1,084$ & $-1,051$ & $-1,312$ & $-1,336$ & $-1,278$ & $-1,217$ & $-1,381$ & $-1,398$ & $-1,344$ & $-1,274$ \\
\hline TIPO ENSAYO & 37 & 38 & 39 & 40 & 41 & 42 & 43 & 44 & 45 & 46 & 47 & 48 \\
\hline $\begin{array}{l}\text { FLECHA } \\
\text { MÁXIMA (metros) }\end{array}$ & $-1,006$ & $-1,028$ & $-0,940$ & $-0,843$ & $-1,187$ & $-1,180$ & $-1,096$ & $-0,927$ & $-1,240$ & $-1,225$ & $-1,146$ & $-0,972$ \\
\hline
\end{tabular}


Figura 6 se muestran los impactos y modelos que producen la flecha máxima y la flecha mínima, respectivamente, en el instante en que se alcanza ésta.

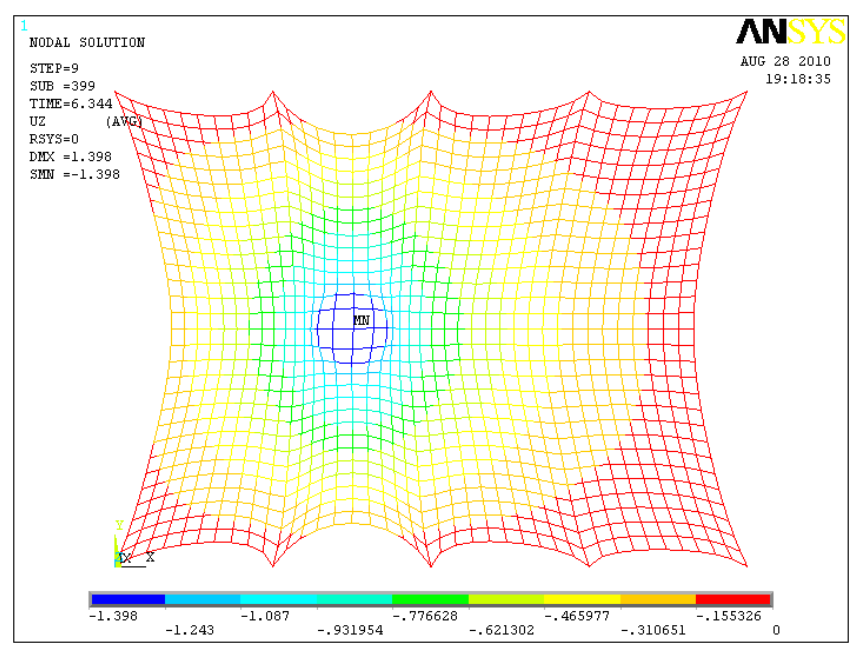

Figura 5. Flecha máxima, modelo 34, valor de 1,398 m.

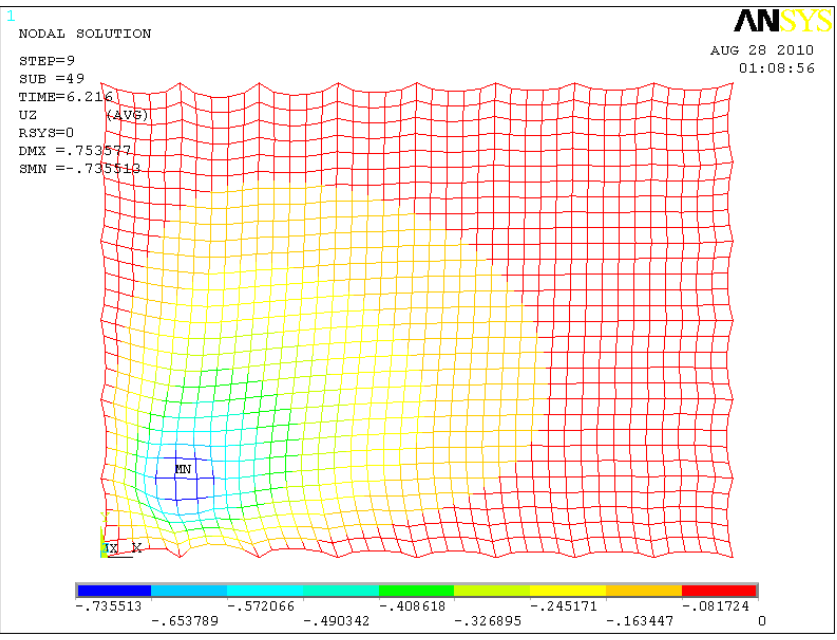

Figura 6. Flecha mínima, modelo 16, valor de 0,736 m.

\subsubsection{Aceleración y Factor de impacto}

El valor máximo de la deceleración sufrida por el accidentado permanece prácticamente constante al aumentar la separación entre anclajes, alcanzando valores de 3-4 g, bastante moderados en relación con las recomendaciones. En el caso de disponer de anclajes laterales, aumentan los máximos ligeramente para las separaciones mayores $(3-5 \mathrm{~g})$.

La Tabla 3 resume los valores máximos y mínimos.
Del análisis de la tabla de resultados de factores de impacto, se observa cómo los ensayos con valores de deceleración máxima y mínima coinciden con los ensayos con valores menores y mayores de flecha respectivamente. El valor máximo para la deceleración es de 4,93 g, obtenido para el impacto de esquina mientras que el valor mínimo alcanza el de 3,23 g en impactos centrados, suficientemente bajos para los valores máximos aceptados en humanos (18).

Las curvas de evolución temporal del factor de impacto son similares en todos los modelos, alcanzando el máximo en instantes previos a la máxima deformación. En la Figura 7 se muestra la historia temporal de la aceleración para el supuesto más desfavorable (ensayo $40^{\circ}$ ).

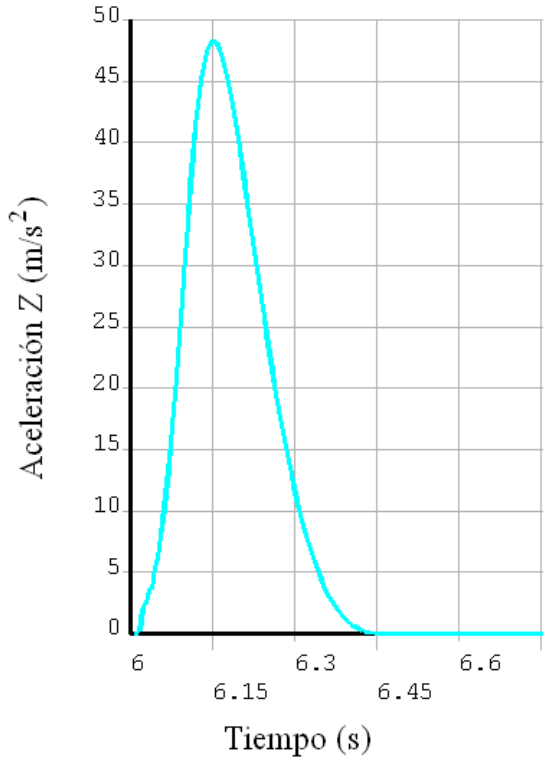

Figura 7. Aceleración Z versus tiempo.

3.1.4. Abertura entre anclajes en el borde inferior de la red

La abertura entre red y borde de forjado no varía prácticamente con la disposición de anclajes laterales. Únicamente se produce variación con el aumento de la separación entre anclajes, duplicándose prácticamente el valor del desplazamiento al duplicar la distancia entre anclajes.

Los puntos de impacto que producen mayores aberturas son los situados junto al borde inferior de la red en la zona entre anclajes. La mayor abertura observada fue de $0,408 \mathrm{~m}$, obtenida para el impacto excéntrico del bloque 3, con laterales libres y separación entre anclajes $100 \mathrm{~cm}$. Este valor podría permitir el paso del accidentado resultando, por tanto, inaceptable. En la Tabla 4 podemos observar los valores en el instante de máximo desplazamiento de la cuerda perimetral.

Tabla 3.

Factores de Impacto sobre lastre.

\begin{tabular}{|c|c|c|c|c|c|c|c|c|}
\hline TIPO & \multicolumn{2}{|c|}{ Bloque 1} & \multicolumn{2}{|c|}{ Bloque 2} & \multicolumn{2}{|c|}{ Bloque 3} & \multicolumn{2}{|c|}{ Bloque 4} \\
\hline ENSAYO & Ensayo $\mathrm{N}^{\circ} 4$ & Ensayo $\mathrm{N}^{\circ} \mathrm{9}$ & Ensayo $\mathbf{N}^{\circ} 16$ & Ensayo $N^{0} 21$ & Ensayo $\mathrm{N}^{\circ} \mathbf{2 8}$ & Ensayo $\mathbf{N}^{0} 33$ & Ensayo $\mathrm{N}^{\circ} 40$ & Ensayo $N^{\circ} 45$ \\
\hline $\begin{array}{l}\text { ACELERACIÓN } \\
\left(\mathrm{m} / \mathrm{seg}^{2}\right)\end{array}$ & 41,09 & 32,50 & 46,31 & 32,31 & 41,00 & 31,67 & 48,33 & 31,94 \\
\hline $\begin{array}{c}\text { FACTOR } \\
\text { DE IMPACTO }\end{array}$ & 4,19 & 3,31 & 4,72 & 3,29 & 4,18 & 3,23 & 4,93 & 3,26 \\
\hline
\end{tabular}


Tabla 4.

Valores de desplazamiento en contorno junto a anclajes.

\begin{tabular}{|c|c|}
\hline & $\sqrt{U x^{2}+U y^{2}+U z^{2}}$ \\
\hline Valores Máximos (m) & 0,17 \\
\hline Bloque 1 & Ensayo $\mathrm{N}^{\circ} 3$ \\
\hline Valores Máximos (m) & 0,171 \\
\hline Bloque 2 & Ensayo $\mathrm{N}^{\circ} 16$ \\
\hline Valores Máximos (m) & 0,408 \\
\hline Bloque 3 & Ensayo $\mathrm{N}^{\circ} 26$ \\
\hline Valores Máximos (m) & 0,406 \\
\hline Bloque 4 & Ensayo $\mathrm{N}^{\mathrm{a}} 38$ \\
\hline
\end{tabular}

La diferencia entre los modelos de los bloques 1 y 2 (anclajes a $50 \mathrm{~cm}$ ) con los de los bloques 3 y 4 (anclajes a $100 \mathrm{~cm}$ ), estriba en el desplazamiento $U z$, movimiento transversal al plano de la red, como consecuencia de la mayor deformación experimentada por las redes con anclajes cada $100 \mathrm{~cm}$. También se observa un mayor desplazamiento en $y$, dirección perpendicular al eje de anclajes. Parece claro que para la abertura perimetral, que supone un riesgo para la caída de personas por el hueco abierto, es determinante la separación entre anclajes.

\subsubsection{Abertura en el borde lateral de la red}

En los casos en que no se dispone de anclajes laterales, el desplazamiento de su cuerda perimetral no se ve afectado por el aumento de la separación entre los demás anclajes. La mayor abertura observada fue de $0,670 \mathrm{~m}$, obtenida en el centro del borde lateral para impacto en posición 12. Cuando la red no tenga continuidad con la del paño vecino, este valor es suficientemente grande como para permitir el paso de una persona de constitución media a través del hueco. Por tanto, si no existe tal continuidad será preciso anclar la red lateralmente.

\subsubsection{Esfuerzos en las trencillas y cuerda perimetral}

El valor máximo de los esfuerzos en las trencillas no varía con la colocación de anclajes en los laterales de la red, aumentando únicamente con la separación entre anclajes, ya que se produce siempre en las trencillas junto a éstos, como efecto local.

En el caso de la cuerda perimetral, la colocación de anclajes laterales cada $50 \mathrm{~cm}$ produce una reducción de los esfuerzos del $55 \%$, bajando la reducción hasta el $17 \%$ al aumentar la separación entre anclajes a $100 \mathrm{~cm}$.

Los puntos de impacto que producen mayores esfuerzos en las trencillas son los situados en la zona central, junto al borde inferior de la red, mientras que para la cuerda perimetral lateral son los situados junto a ella y, dependiendo de la pre- sencia de anclajes laterales o no, su zona central o la esquina respectivamente.

El valor más alto del esfuerzo en la cuerda perimetral fue de $2261 \mathrm{~N}$, registrado en el tramo de esquina con impacto en posición 8 , anclajes a $100 \mathrm{~cm}$ y laterales libres. Para las trencillas, el valor máximo fue de $647 \mathrm{~N}$, registrado con impacto en posición 1 y anclajes a $50 \mathrm{~cm}$ en los cuatro bordes. En la Tabla 5 se muestran los máximos valores en trencillas y cuerdas perimetrales.

Estos valores son inferiores a los indicados por Saiz (7), donde se citan valores experimentales para la resistencia de rotura de las trencillas de $1156 \mathrm{~N}$, incluida la reducción correspondiente a la presencia del doble nudo de telar.

\subsection{Redes de paño pequeño}

\subsubsection{Geometría de la malla}

Existe sensibilidad ante el cambio de geometría, de malla cuadrada (Q) a malla en rombo (D). Las flechas máximas de los modelos con mallas en rombo presentan valores un $12 \%$ menores que los modelos con mallas cuadradas existiendo una correlación lineal entre ambos grupos de deformaciones para diferentes valores de rigidez de trencilla. En la red en rombo parece que la respuesta es más inmediata y esta celeridad en la reacción puede generar menores deformaciones punta y menores reacciones punta. A su vez, esta inmediatez se justifica por la respuesta de las trencillas diagonales centrales que apoyan directamente en los puntos de anclaje.

\subsubsection{Rigidez de la trencilla}

La modificación del módulo de deformación de la trencilla presenta la sensibilidad esperada (se aumenta la rigidez del conjunto). Se observa una disminución de la flecha y un aumento de la aceleración máxima del lastre con el aumento de rigidez. La reacción en los apoyos de la red muestra una relación cuasi lineal (Figura 8).

\subsubsection{Excentricidad de la carga}

En cuanto a la flecha máxima, la excentricidad de la carga para redes pequeñas no provoca diferencias significativas. Analizando distintos modelos se ha observado una variación en la flecha, relativa a este parámetro, menor al 15\% del valor máximo alcanzado. En la Figura 9 se observa la flecha máxima para un impacto centrado y en la Figura 10 para uno excéntrico. Para el mismo modelo de red, la diferencia de flecha supone una disminución del 13,44\% respecto al valor máximo.

Tabla 5 .

Esfuerzos en trencillas y cuerdas perimetrales.

\begin{tabular}{|c|c|c|c|}
\hline & Máximo axil trencillas & Máximo axil trencilla borde & Máximo axil cuerda perimetral \\
\hline Valores Máximos (N) & 529,4 & 317,32 & 2411 \\
\hline Bloque 1 & Ensayos $\mathrm{N}^{\circ} 1$ & Ensayos $\mathrm{N}^{\mathrm{o}} 3$ & Ensayo $N^{\circ} 12$ \\
\hline Valores Máximos $(\mathrm{N})$ & 537,41 & 306,45 & 870,61 \\
\hline Bloque 2 & Ensayo $\mathrm{N}^{\mathrm{o}} 14$ & Ensayo $\mathrm{N}^{\circ} 15$ & Ensayo $\mathrm{N}^{\mathrm{O}} 2 \mathrm{O}$ \\
\hline Valores Máximos (N) & 646,86 & 460,09 & 2260,6 \\
\hline Bloque 3 & Ensayo $\mathrm{N}^{\circ} 25$ & Ensayo $\mathrm{N}^{\circ} 26$ & Ensayo $\mathrm{N}^{\circ} 32$ \\
\hline Valores Máximos (N) & 643,84 & 482,72 & 1867,9 \\
\hline Bloque 4 & Ensayo $\mathrm{N}^{\mathrm{a}} 37$ & Ensayo $\mathrm{N}^{\mathrm{a}} 4 \mathrm{O}$ & Ensayo $\mathrm{N}^{\circ} 40$ \\
\hline
\end{tabular}




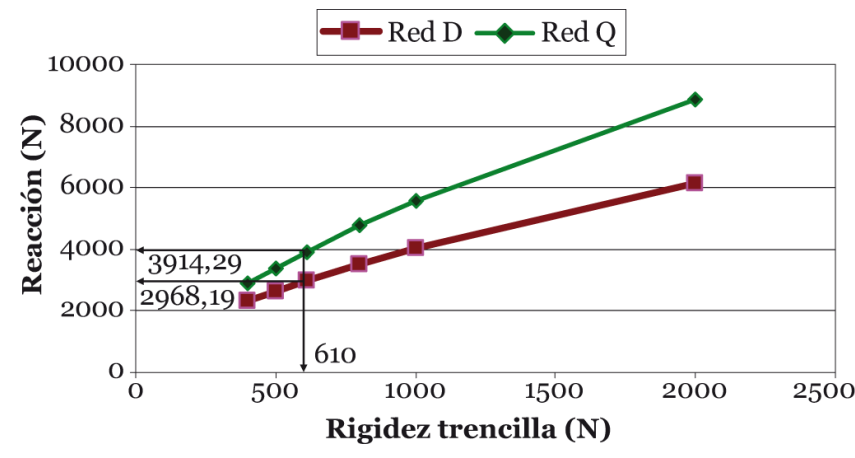

Figura 8. Sensibilidad de las reacciones frente a la rigidez de las trencillas.

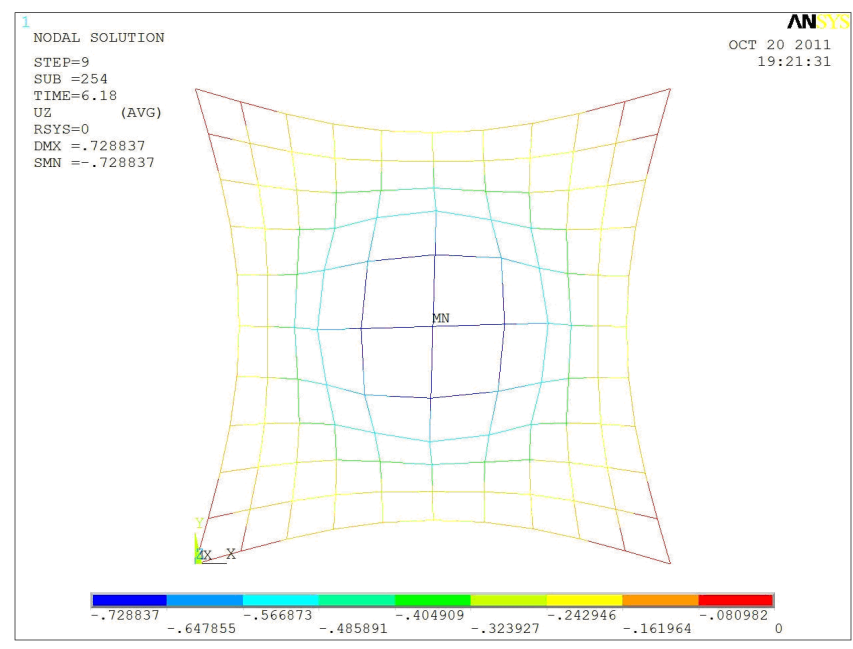

Figura 9. Flecha por impacto centrado, red pequeña, valor de 0,729 $\mathrm{m}$.

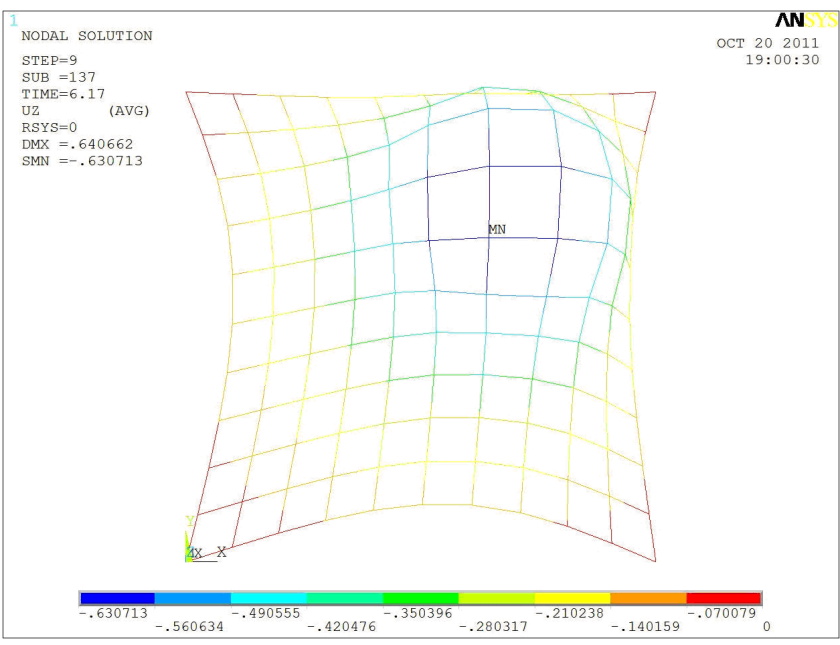

Figura 10. Flecha por mpacto excéntrico, red pequeña, valor de $0,631 \mathrm{~m}$.

La excentricidad de la carga es un parámetro muy sensible en los modelos para las medidas de las reacciones en los apoyos y los esfuerzos en las cuerdas perimetrales. Frente al impacto central, la máxima excentricidad provoca incrementos del $45 \%$ en el esfuerzo axil máximo de la cuerda perimetral. Además, la excentricidad provoca una modificación en la composición de la fuerza de la reacción, aumentando el valor $Z$ (dirección perpendicular a la red), disminuyendo la componente de la dirección de la excentricidad y aumentando el conjunto. La respuesta ante un impacto excéntrico genera una reacción mayor y más rápida, ya que se transfieren las fuerzas del impacto con mayor celeridad al anclaje.

\subsubsection{Cuerdas perimetrales}

Para las cuerdas perimetrales, en los modelos con cables de acero en los perímetros horizontales se observa una reducción de la flecha independiente de la rigidez de la trencilla (Tabla 6). El factor de impacto muestra tendencias opuestas (Tabla 7) con cable de acero en el perímetro horizontal en las redes $\mathrm{Q}$ (diferencia creciente) frente a las redes $\mathrm{D}$ (diferencia decreciente). Esto puede ser debido a la influencia del perímetro en la rigidez del conjunto, siendo menor en la geometría en rombo por la misma razón sugerida en 3.2.1. En todo caso, este factor alcanza valores considerablemente superiores a los alcanzados en paños grandes. Las reacciones en los apoyos quedan distorsionadas por la constitución de perímetros con cables de acero, éstas elevan los valores considerablemente (10 veces). Las aberturas perimetrales dependen de las cuerdas perimetrales; con cables de acero son prácticamente nulas debido a su bajísima flecha y con cuerdas perimetrales textiles, en el peor de los casos, alcanzan valores de $0,20 \mathrm{~m}$, espacio insuficiente para suponer riesgo de caída para una persona.

\subsubsection{Tensión inicial de la red}

La tensión inicial en los modelos muestra dos características, una más significativa que la otra. La primera, esperada, es la menor deformación máxima en los modelos con tensión inicial. La segunda, inesperada, una ligera tendencia decreciente en el factor de impacto (aceleración máxima sufrida por el lastre). La menor deformación máxima vendría explicada por la mayor rigidez del sistema al estar en carga y el menor factor de impacto podría explicarse por la mayor rigidez inicial del sistema en carga. Las mayores aceleraciones iniciales que recibe el lastre reducen su pico (Figura 11). En los modelos con tensión inicial, se alcanza antes la flecha máxima, que es menor, curiosamente con menor factor de impacto.

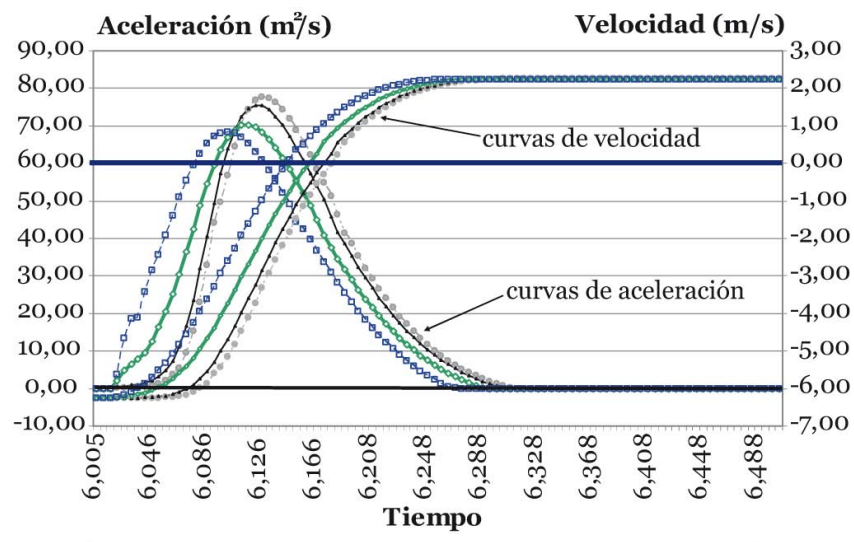

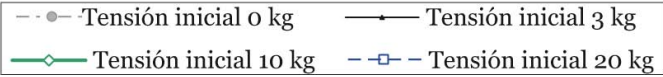

Figura 11. Factor de impacto con cable o cuerda perimetral.

\section{CONCLUSIONES}

Las redes verticales en fachada son una solución posible para evitar caídas de altura al personal durante la construcción de edificios, en los trabajos entre pisos de los oficios, una vez aca- 
Tabla 6.

Flechas máximas con cable o cuerda perimetral

\begin{tabular}{|c|c|c|c|c|c|c|}
\hline \multirow{2}{*}{$\begin{array}{c}\text { Módulo } \\
\text { de deformación } \\
(\mathbf{N})\end{array}$} & \multicolumn{4}{|c|}{ Flecha máxima (m) / UZ Nodo 5o } \\
\cline { 2 - 7 } & Con cable & Sin cable & Diferencia & Con cable & Sin cable & Diferencia \\
\cline { 2 - 7 } & 0,566 & 0,687 & 0,121 & 0,578 & 0,639 & 0,062 \\
\hline 610 & 0,409 & 0,533 & 0,123 & 0,416 & 0,461 & 0,044 \\
\hline 2000 & \multicolumn{3}{|c}{} \\
\hline
\end{tabular}

Tabla 7.

Factor de impacto con cable o cuerda perimetral

\begin{tabular}{|c|c|c|c|c|c|c|}
\hline \multirow{2}{*}{$\begin{array}{c}\text { Módulo } \\
\text { de deformación } \\
\text { (N) }\end{array}$} & \multicolumn{4}{|c|}{ Factor de impacto (g) } \\
\cline { 2 - 7 } & Con cable & Sin cable & Diferencia & Con cable & Sin cable & Diferencia \\
\cline { 2 - 7 } & ConADRADA & 7,545 & 0,840 & 6,661 & 6,317 & $-0,344$ \\
\hline 610 & 6,705 & 13,673 & 2,298 & 10,899 & 10,036 & $-0,863$ \\
\hline
\end{tabular}

bada la estructura o en fases de ejecución de fachadas. Para este ámbito de trabajo, la energía cinética con que el posible accidentado llega a la red es moderada y el uso de tales redes, incluso de pequeño tamaño, que resultan más rígidas que otros tipos, genera factores de impacto aceptables sobre él.

Los anclajes a la estructura en este tipo de redes resultan fundamentales. Por ello, determinar las reacciones es imprescindible para avanzar en su configuración. Las reacciones vienen condicionadas por la distancia entre puntos de anclaje y su configuración, así como por la rigidez de la cuerda perimetral o de la red. El incremento de la rigidez del conjunto supone mayor fuerza de reacción y, por tanto, requiere una mejor distribución de puntos anclaje en el perímetro. En redes de paño pequeño, se ha comprobado el efecto positivo del tipo de geometría de la malla en rombo en la reducción de la reacción.

La flecha máxima alcanzada por la red varía entre 0,75 y 1,5 m, dependiendo del punto de impacto. Para las redes pequeñas la flecha alcanza el menor de dichos valores. Se evidencia la relación directa entre máxima flecha y menor factor de impacto, y viceversa. Las mayores flechas las encontramos en impactos centrados, donde la red puede desarrollar la máxima deformación, por lo que amortigua mejor el impacto reduciendo la deceleración máxima sufrida por el lastre.

Las deceleraciones sufridas por el potencial accidentado están en torno a 4-5 g en el caso de redes grandes de todo el paño de fachada, y en torno a 7-8 g para las pequeñas de 1 $\mathrm{m}^{2}$. Los factores de impacto dependen de la excentricidad del impacto, de la red y, en menor medida, del tensado inicial, del tipo de geometría y de las cuerdas perimetrales.
Los resultados muestran que las tensiones en la red y en las cuerdas perimetrales son aceptables para las redes de seguridad corrientemente usadas en otros sistemas. Lo mismo cabe decir respecto de los esfuerzos sobre los anclajes (exceptuando el caso de los cables de acero).

Los modelos han permitido comprobar que para redes grandes la separación máxima entre anclajes perimetrales inferiores (o laterales extremos) es de $50 \mathrm{~cm}$, generándose aberturas peligrosas para mayores separaciones. Por el contrario, para redes pequeñas de $1 \times 1 \mathrm{~m}^{2}$ es suficiente con anclar a las cuatro esquinas, sin que se produzcan aberturas peligrosas.

Para las redes pequeñas se ha comprobado que un cierto pretensado previo conduce a menores flechas, pero curiosamente también a menores factores de impacto, al variar la distribución temporal de las aceleraciones. Este efecto debe ser estudiado con mayor detenimiento, así como la influencia de otros factores como la geometría de la malla en rombo.

Más allá de las investigaciones realizadas, sería conveniente el análisis del sistema de anclaje dada su importancia relativa en el fallo del sistema. Igualmente, también sería deseable la realización de nuevas simulaciones con otros ángulos de incidencia sobre la red, y de algunos ensayos reales con instrumentación de medida, para confirmar la bondad cuantitativa de las conclusiones de estos modelos numéricos. No obstante, y aún en su ausencia, los resultados obtenidos pueden ser muy útiles y servir de orientación al grupo de trabajo encargado de redactar la norma reguladora.

\section{REFERENCIAS}

(1) Consejo Europeo. (1989). Directiva 89/391/CEE del Consejo, de 12 de junio de 1989, relativa a la aplicación de medidas para promover la mejora de la seguridad y de la salud de los trabajadores en el trabajo. Diario Oficial, $\mathrm{n}^{\circ} \mathrm{L} 183$ de 29/06/1989 pp.0001-0008.

(2) Cortes Generales. (1995). Ley 31/1995 de Prevención de Riesgos Laborales. Boletín Oficial del Estado, nº 269, (España).

(3) Ministerio de la Presidencia. (1997). R. D. 1627/1997 de 24 de octubre de 1997 por el que se establecen disposiciones mínimas de seguridad y de salud en las obras de construcción. Boletín Oficial del Estado, n⿳0 256, (España).

(4) AENOR-CEN. (2004). UNE-EN 1263 Redes de Seguridad. Parte 1: Requisitos de seguridad, métodos de ensayo. Asociación Española de Normalización (AENOR).

(5) AENOR-CEN. (2004). UNE-EN 1263 Redes de Seguridad. Parte 2: Requisitos de seguridad para la instalación de redes de seguridad. Asociación Española de Normalización (AENOR). 
(6) AENOR-CEN. (2013). UNE-EN 13374 Sistemas provisionales de protección de borde. Especificaciones del producto, método de ensayo. Asociación Española de Normalización (AENOR).

(7) Saiz, J., Irles, R., Arcenegui, G.A., Naharro, M. (1997). Las Redes de Seguridad en la Construcción. Univ. de Alicante D.G.T. (Generalitat Valenciana). Alicante (España).

(8) Segovia, E. (2005). Criterios para el diseño de los sistemas anticaída utilizados en construcción y sometidos a impacto. (Tesis Doctoral) Univ. Politécnica de Valencia. Dtº Mecánica de Medios Continuos y Teoría de Estructuras.

(9) Riquelme-Moya, P., Romero-Flores, S., Irles-Mas, R., Segovia-Eulogio, E. (2011). Redes de seguridad verticales en fachada. Workshop - Investigación e innovación en protecciones colectivas y medios auxiliares de edificación, Madrid.

(10) Riquelme-Moya, P. (2010). Análisis de un modelo numérico para redes pequeñas de seguridad en construcción: parámetros de diseño (Trabajo de investigación para diploma de estudios avanzados). Universidad de Alicante.

(11) Romero-Flores, S. (2010). Análisis del comportamiento frente al impacto en redes de seguridad de cierre vertical de paño grande (Trabajo de investigación para diploma de estudios avanzados). Universidad de Alicante.

(12) Pomares-Torres, J.C., Irles-Mas, R., Segovia-Eulogio, E. (2011). Personal protection rails for strong impacts. 4th International Conference on Safety and Security Engineering, Amberes (Bélgica).

(13) Romero-Flores, S., Riquelme-Moya, P., Irles-Mas, R., Segovia-Eulogio, E. (2011). Floor to ceiling vertical safety nets. $4^{\text {th }}$ International Conference on Safety and Security Engineering, Amberes (Bélgica).

(14) Irles, R., González, A., Segovia, E., Maciá, A. (2002). Las redes verticales de seguridad en la construcción de edificios I. Informes de la Construcción, 53(477): 21-29, doi: http://dx.doi.org/10.3989/ic.2002.v53.i477.639.

(15) Irles, R., González, A., Segovia, E., Maciá, A., Pomares, J. C. (2007). Las redes verticales de seguridad en la construcción de edificios II. Informes de la Construcción, 59(505): 37-51, doi: http://dx.doi.org/10.3989/ic.2007.v59.i505.499.

(16) Irles, R., Maciá, A, Segovia, E., Saiz, J. (1998). Redes verticales de seguridad. Resultados teóricos y experimentales. III European Forum in Science, Security and Health, Oviedo (España).

(17) Arcenegui, G., Saiz, J., Irles, R., Naharro, M. (1998). Les Filets de Sécurité sur supports de type potence. Int. Fall Protection Symposium, Wuppertal (Alemania).

(18) Sulowski, A. C. (2006, 14-15 de junio). How good is the 8kN Maximum Arrest Force limit in industrial Fall Arrest Systems? Int. Fall Protection Symposium, Seattle, WA.

(19) Paureau, E., Parisot, E., Schuler, B. (1989). Filets en grandes nappes. Eficacité, performances, règles de pose. Cahiers de notes documentaires $137(4 \mathrm{e}): 655-675$. 